\title{
Gutta Percha, Rubber or Balata? Establishing Analytical Markers for Identification of Cultural Heritage Materials
}

\author{
Hanna M. Szczepanowska ${ }^{1}$, and C. Velson Horie ${ }^{2}$ \\ ${ }^{1}$ National Heritage Board, Heritage Conservation Centre, Singapore \\ ${ }^{2}$ Collection Care and Conservation Consultant, Manchester, UK
}

Gutta percha (GP), natural rubber (NR) and balata (B) were innovative plant products that resulted from imperialist exploration, enabling the transformation of $19^{\text {th }}$ century industry and politics. Their introduction in the first half of the $19^{\text {th }}$ century prompted many experiments in how they could be applied in diverse cultural heritage artefacts. Gutta percha (GP) was used in dentistry, undersea telegraph cables, and elegant cases for daguerreotypes. NR was used for waterproof clothing (Macintoshes and Wellington boots) and tyres for increasingly important automobile industries, as well as a wealth of industrial and domestic products. Balata could be added as mixtures in GP and NR products. The base polymeric materials have identical chemical formulae; all are polyisoprenes differing only in their molecular structure. In the past, the polymers of GP and B were said to be trans-1,4 polyisoprene, whereas NR is cis-1,4 polyisoprene. This structural difference affects their physical properties significantly. GP and balata are both thermoplastics, which can be moulded when hot then set to a hard solid. In contrast, NR can be stretched, then will snap back into its previous shape, a property that is improved enormously by cross-linking (vulcanisation). Both polyisoprenes are highly unsaturated hydrocarbons, very susceptible to oxidation, resulting in degradation and change of properties. The chemical nature of "polymer" was unknown until well into the $20^{\text {th }}$ century, so early workers used entirely empirical methods to extract, modify and manipulate the raw materials. As a result, many unpredictable impurities (original or added) may be found in the resulting products.

The aim of this study is to establish microanalytical markers in GP, NR and balata that can guide their identification in cultural heritage. This investigation identifies chemical alterations (using Raman spectroscopy) over time in raw latices, coagulated solids and historic samples in Southeast Asia. Once established, the set of diagnostic features characteristic for GP, NR and B will be applied to the analysis of actual museum artefacts.

Three sets of samples were analyzed: (1) raw, liquid latex of GP, NR and balata, 8 samples, (2) 1980-1986, 8 samples of GP, and (3) 1892-1906, 10 samples of GP, NR and balata. Raman spectra were recorded using Renishaw, InVia Qontor spectrometer, with 785nm laser, 1200 line grating, in the range $100-3500 \mathrm{~cm}-1 \mathrm{~cm}$, using detector Renishaw Centrus OC4E55. The surface morphology was studied using Confocal Laser Scanning Microscope (CLSM), Olympus LEXT, with 405nm laser source, average number of scans 9004000, captured with CCD camera, 16.6-pixelscan, $0,03 \mu \mathrm{m}$ per pixel.

Figure 1. The Raman spectra of fresh latices of GP, NR and B were similar, reflecting their common base polymer polyisoprene, but revealed the differences typical of trans-isomers (GP) and cis- (NR). Strong peaks in the region $1680-1650 \mathrm{~cm}^{-1}$, which are characteristic of trans-isomers absorbing in the higher wave numbers than cis-isomers were also present in the NR latex. Other peaks characteristic of trans-isoprene, such as 1380 and in the region of $1440-1480 \mathrm{~cm}^{-1}$ are prominent in the spectra of GP. Figure 2 . The greatest difference between GP, NR and B is in the region of $2830-2900 \mathrm{~cm}^{-1}$ typical for trans- (saturated) isomers, present in GP and NR and missing in B, which raises doubts about previous proposals of its composition. These spectra of raw materials include contributions from many other natural resins, proteins etc. These contributions complicate the analysis, but provide a method to elucidate mixtures present in historic objects. Figure 3. The surface morphology and topography of fresh lattices coagulated on glass slides was visualized 
with CLSM to show differences between each raw material. It starts a long term observation process. Our preliminary data shows that spectrometry may be a valuable technique in establishing unique markers that can be used in identifying presence of GP, NR and B in artefacts. Further work will involve using FTIR-ATR and $\mathrm{PyrGC} / \mathrm{MS}$ to define more closely their chemical signatures. The discrepancies and variability discovered will contribute to a better understanding of this historically important and little analyzed material, enabling better preservation of cultural heritage objects made of these complex natural products.

\section{References:}

[1] Langenheim, J.H. 2003. Plant Resins: Chemistry, Evolution, Ecology and Ethnobotany. Timber Press.

[2] Clouth, F. 1903. Rubber, Gutta-Percha and Balata, MacLaren \& Sons and D. van Nostrand.

[3] Williams, L. Economic Botany 18 (1964), p. 5.

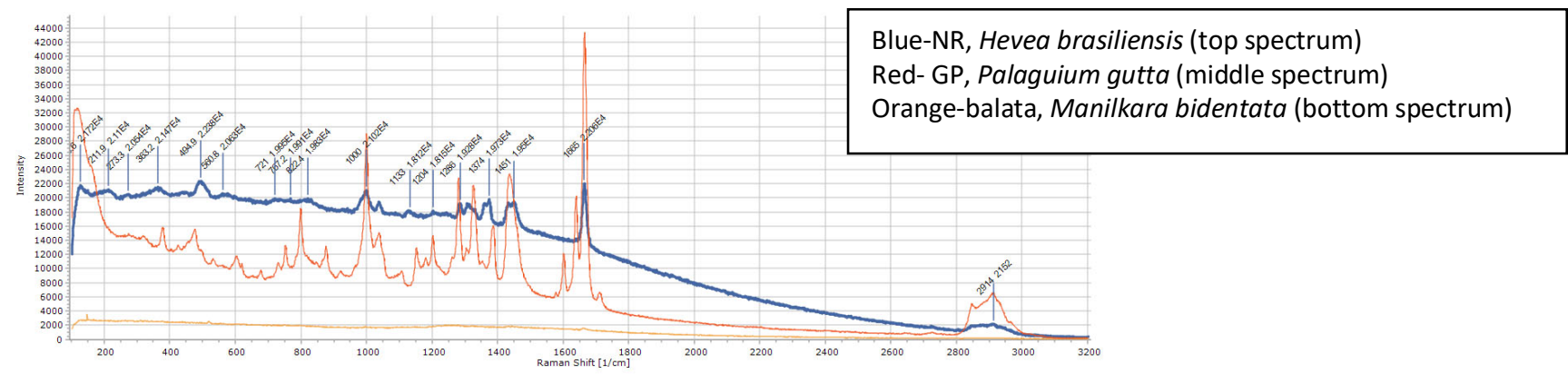

Figure 1. From Set 1, Raman spectra from three fresh latices. The fingerprint for Raman spectra is dominated by strong peaks at 1450-1050 $\mathrm{cm}^{-1}$, with common peaks for GP and B: 1387 and 1326, 1280, 1201 and $1151 \mathrm{~cm}^{-1}$ and absent in the spectra of NR.

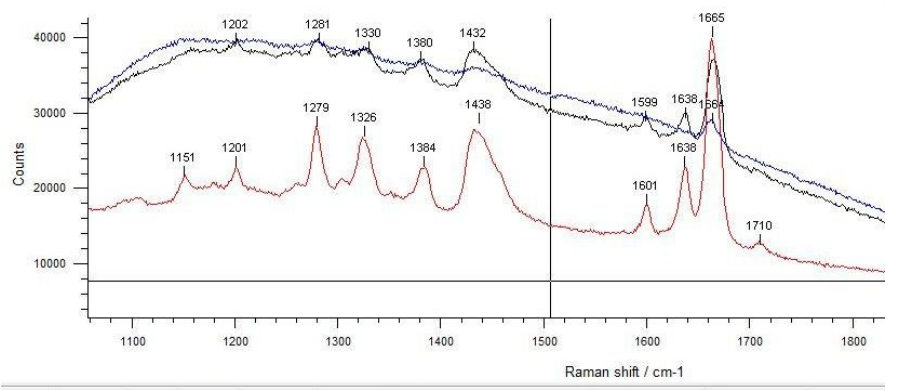

Blue-40-year old sample of $P$. gutta (top spectrum)

Black-coagulant of P.gutta (middle spectrum)

Red-fresh latex, $P$. gutta (bottom spectrum)

Figure 2. From Sets 1-3, Raman spectra from GP latex, coagulant solidified during harvest and a 40-year old material, all from the Lingga Isl. The three spectra show the trans-polyisoprene peaks in the region of interest and may be potential markers for the identification of GP in artefacts.

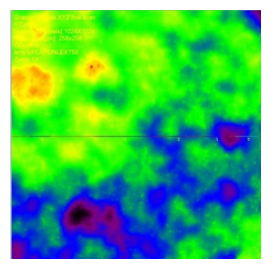

\section{$3 \mathrm{~A}$}

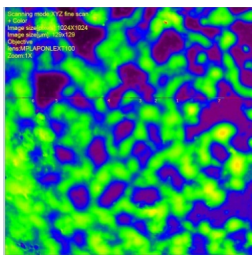

3B

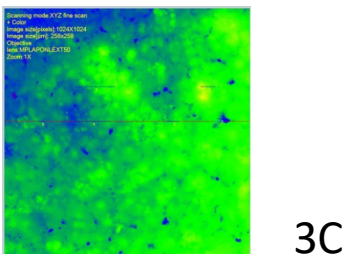

Figure 3. The surface topography of fresh lattices from Set 1 visualized as intensity maps using CLSM, area

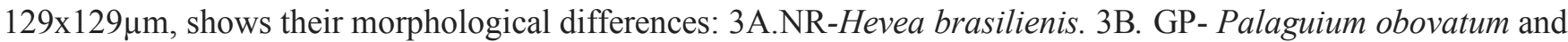
3C. Balata- Manilkara bidentata. 